\title{
Intra- and inter-operator reproducibility of US point shear-wave elastography in various organs: evaluation in phantoms and healthy volunteers
}

\author{
Riwa Kishimoto $^{1}$ D $\cdot$ Katsuhiko Kikuchi $^{1,2} \cdot$ Atsuhisa Koyama $^{3} \cdot$ Jeff Kershaw $^{1} \cdot$ Tokuhiko Omatsu $^{1}$. \\ Yasuhiko Tachibana $^{1} \cdot$ Mikio Suga $^{3} \cdot$ Takayuki Obata $^{1}$
}

Received: 11 January 2019 / Revised: 18 March 2019 / Accepted: 21 March 2019/Published online: 14 May 2019

(C) The Author(s) 2019

\begin{abstract}
Purpose This study was conducted in order to assess the intra- and interoperator reproducibility of shear-wave speed (SWS) measurement on elasticity phantoms and healthy volunteers using ultrasound-based point shear-wave elastography.

Materials and methods This study was approved by the institutional review board. Two operators measured the SWS of five elasticity phantoms and seven organs (thyroid, lymph node, muscle, spleen, kidney, pancreas, and liver) of 30 healthy volunteers with 1.0-4.5 MHz convex (4C1) and 4.0-9.0 MHz linear (9L4) transducers. The phantom measurements were repeated ten times, while the volunteer measurements were performed five times each. Intra- and interoperator reproducibility was assessed. Interoperator reproducibility was also evaluated with the $95 \%$ Bland-Altman limits of agreement (LOA).

Results In phantoms, all intraclass correlation coefficients (ICCs) were above 0.90 and the $95 \%$ LOA between the two operators were less than $\pm 18 \%$. In volunteers, intraoperator ICCs were $>0.75$ for all regions except the pancreas. Interoperator ICC was above 0.75 for the right lobe of the liver (depth $4 \mathrm{~cm}$ ) and the kidney, but the $95 \%$ LOA was less than $\pm 25 \%$ only for the liver. Conclusion Although excellent in phantoms, interoperator reproducibility was insufficient for all regions in the volunteers other than the right hepatic lobe at a depth of $4 \mathrm{~cm}$. Clinicians should be aware of the $95 \%$ LOA when using SWS in patients.

Key Points

- Our phantom study indicated a high reproducibility for shear-wave speed (SWS) measurements with point shear-wave elastography ( $p S W E)$.

- In volunteers, intraoperator reproducibility was generally high, but the interoperator reproducibility was not high enough except for the right hepatic lobe at $4 \mathrm{~cm}$ depth.

- To evaluate interoperator reproducibility, the 95\% limits of agreement (LOA) between operators should be considered in addition to the intraclass correlation coefficient (ICC).
\end{abstract}

Keywords Elasticity imaging techniques · Observer variation · Reproducibility of results · Ultrasonography

Electronic supplementary material The online version of this article (https://doi.org/10.1007/s00330-019-06195-8) contains supplementary material, which is available to authorized users.

Riwa Kishimoto

kishimoto.riwa@qst.go.jp

1 National Institute of Radiological Sciences, National Institutes for Quantum and Radiological Science and Technology, 4-9-1 Anagawa, Chiba 263-8555, Japan
2 Tokyo-Kita Medical Center, 4-17-56, Akabanedai, Tokyo 115-0053, Japan

3 Center for Frontier Medical Engineering, Chiba University, 1-33, Yayoi-chou, Chiba 263-8522, Japan 


$\begin{array}{ll}\text { Abbreviations } \\ \text { 2D SWE } & \text { 2D color-coded shear-wave elastography } \\ \text { ARFI } & \text { Acoustic radiation force impulse } \\ \text { BMI } & \text { Body mass index } \\ \text { ICC } & \text { Intraclass correlation coefficient } \\ \text { IQR } & \text { Interquartile range } \\ \text { LOA } & \text { Limits of agreement } \\ \text { pSWE } & \text { Point shear-wave elastography } \\ \text { QIBA } & \text { Quantitative Imaging Biomarkers Alliance } \\ \text { ROI } & \text { Region of interest } \\ \text { SWS } & \text { Shear-wave speed } \\ \text { TE } & \text { Transient elastography } \\ \text { US } & \text { Ultrasound } \\ \text { VTQ } & \text { Virtual touch quantification }\end{array}$

\section{Introduction}

Ultrasound (US) elastography has been widely used to evaluate liver fibrosis or to differentiate between benign and malignant lesions in breast, thyroid, and prostate [1-4]. Measurements of shear-wave speed (SWS) provide quantitative information about organ stiffness, which is a potential noninvasive biomarker [5]. The Quantitative Imaging Biomarkers Alliance (QIBA) organized by the Radiological Society of North America has selected SWS as a potential biomarker. Currently, efforts are underway to make a profile or an implementation guide to achieve sufficient accuracy and avoid variability in the measurement [6].

There are three methods for SWS measurement: (i) transient elastography (TE), which is $1 \mathrm{D}$ elastography without an anatomic B-mode image guide [7]; (ii) point shear-wave elastography (pSWE), which provides single-point measurement within a B-mode image; and (iii) 2D color-coded shearwave elastography (2D SWE), which produces $2 \mathrm{D}$ colorvelocity maps and allows for multiple measurements to be obtained. pSWE and 2D SWE apply the acoustic radiation force impulse (ARFI) technique, which is based on the emission of short-duration acoustic pulses into tissues that induce localized tissue displacement, resulting in shear-wave propagation away from the region of excitation [8-10].

While TE has been standardized and validated in numerous centers worldwide, its application is limited to organs directly beneath the transducer, and it is seldom used outside the liver, spleen, and kidney. On the other hand, although the main clinical applications of pSWE and 2D SWE are to evaluate liver fibrosis [11-13] and breast [14] and thyroid nodules [15], they can also be applied for the evaluation of inflammatory and neoplastic diseases in the pancreas [16], kidney [17, 18], prostate [19], lymph node [20], and muscles [21]. Many studies have evaluated the accuracy of SWS measurement as a biomarker for staging liver fibrosis or differentiating a malignant lesion from a benign one $[8,9,11,14-16,18,20]$. SWS is also used as a noninvasive biomarker for assessing the therapeutic response of antifibrotic, antiviral, or anticancer drugs [22, 23]. Before SWS can be reliably used as a biomarker for clinical diagnosis, it is essential to estimate the reproducibility of measurements. To date, however, other than for the measurement of liver stiffness [24], the number of studies that specifically address the intra- and interoperator reproducibilities of SWE is limited $[25,26]$.

The aim of this study is to examine the intra- and interoperator reproducibility of SWS measurement with pSWE in elasticity phantoms and the organs of healthy volunteers.

\section{Materials and methods}

\section{Subjects}

\section{Phantoms}

Five rectangular parallelepiped phantoms $\left(9 \times 13 \times 13 \mathrm{~cm}^{3}\right)$, suitable for MR and US elastography, were made. These were acrylamide-based homogeneous phantoms containing graphite particles [27]. The storage moduli were measured with a rheometer, and as SWS is proportional to the square root of the stiffness, the corresponding SWSs of the phantoms were also calculated (Table 1). Phantom homogeneity was confirmed with MR elastography, and the corresponding SWSs were also estimated (Table 1) [27].

\section{Healthy volunteers}

This study was approved by the institutional review board and written informed consent was obtained from all participants.

Thirty healthy volunteers (female: male $=15: 15$, mean age 38.5 years, range $21-58$ years; mean body mass index (BMI), $21.7 \mathrm{~kg} / \mathrm{m}^{2}$, range $16.7-29.5 \mathrm{~kg} / \mathrm{m}^{2}$ ) without a history of liver, pancreatic, renal, or thyroid disease were included in this study. The volunteers were asked to fast for at least $4 \mathrm{~h}$ prior to examination.

\section{Imaging techniques}

An Acuson S2000 US system (Siemens Healthineers) with 1.0-4.5 MHz convex (4C1) and 4.0-9.0 MHz linear (9L4) transducers was used. SWS measurements were obtained with the virtual touch quantification (VTQ) software, which is Siemens' implementation of the pSWE method, utilizing the ARFI technique. The region of interest (ROI) was $10 \times 6 \mathrm{~mm}^{2}$ for the $4 \mathrm{C} 1$ transducer and $5 \times 5 \mathrm{~mm}^{2}$ for the 9L4 transducer. The room temperature was maintained at $20^{\circ} \mathrm{C}$.

Examinations were performed by two operators in separate sessions. Each observer made the required number of measurements for a phantom or organ in successive measurements over a period of 1 or 2 min with minimum movement of the 
Table 1 Storage modulus and shear-wave speed of five phantoms measured with a rheometer and MR elastography

\begin{tabular}{lllllc}
\hline Storage modulus (kPa) & 2.1 & 5.2 & 9.7 & 13.3 & 25.0 \\
SWS (m/s) & 1.41 & 2.23 & 3.01 & 3.56 & 4.86 \\
SWS w/ MRE (m/s) & $1.72 \pm 0.05$ & $2.42 \pm 0.01$ & $3.31 \pm 0.02$ & $3.75 \pm 0.05$ & $4.03 \pm 0.04$ \\
\hline
\end{tabular}

$S W S=$ shear-wave speed, $M R E=$ MR elastography transducer, then moved on to the next phantom or organ. For the phantom studies, one operator was a board-certified radiologist and the other a graduate student of medical engineering. Each operator measured the SWS in a ROI of the same area at four different depths $(2,4,6$, and $8 \mathrm{~cm}$ with the $4 \mathrm{C} 1$ transducer and 1,2,3, and $4 \mathrm{~cm}$ with the 9L4 transducer) for each phantom. The measurements were repeated ten times at each depth. Because the hardest phantom was outside of the measurable range of the $4 \mathrm{C} 1$ transducer, it was measured only with the 9L4 transducer. Occasionally, SWS measurement was unreliable or out of range (i.e., SWS is displayed as "x.xx m/s" on this system), which was taken to indicate measurement failure. Attempts were made to obtain ten valid measurements, but when the failure rate, which was calculated as the number of measurement failures divided by the number of measurements, was more than $50 \%$, the data was excluded from further evaluation.

For the volunteer studies, a board-certified radiologist and a registered medical sonographer measured SWS. Both had more than 20 years of experience in ultrasound examination. The operators measured the SWSs for seven organs: left lobe of the thyroid, cervical lymph node, right brachioradialis muscle, spleen, left kidney, pancreas, and liver (Fig. 1). For the liver, SWS measurements were obtained in the right lobe at three different depths $(4,6$, and $8 \mathrm{~cm})$, while only one measurement was made in the left lobe. In accordance with the results of the phantom study and the minimum measurement number described in the guidelines of the World Federation for Ultrasound in Medicine and Biology [1], measurements were repeated five times in each region for the volunteer study. Thus, a total of 100 SWSs ( 2 operators $\times 10$ regions $\times 5$ times $)$ were obtained for each volunteer. Examination was performed with the 9L4 transducer for the thyroid, lymph node, and brachioradialis muscle, and the 4C1 transducer was used for the spleen, left kidney, pancreas, and liver. The volunteers were placed in a supine position and the SWSs of the abdominal organs were measured during breath-holding after subtle inspiration. The spleen, left kidney, and right hepatic lobe were scanned with an intercostal approach, while the pancreas and left hepatic lobe were scanned with a subcostal approach. Care was taken not to include any blood vessels or biliary structures. For the kidney, the operators were asked to put the ROI in the renal cortex to avoid the medulla. SWSs were measured parallel to the long axis for the lymph node and muscle and perpendicular for the thyroid (Fig. 1).

\section{Statistical analysis}

For the volunteer study, the within-population interquartile range (IQR) was estimated to assess the heterogeneity of the population.

To evaluate intraoperator reproducibility, the one-way random, absolute agreement, average-measure ICC (intraclass correlation coefficient) was calculated using both five and ten measurements for the phantom study (ICC $(1,5)$ or $(1,10))$, and using all five measurements
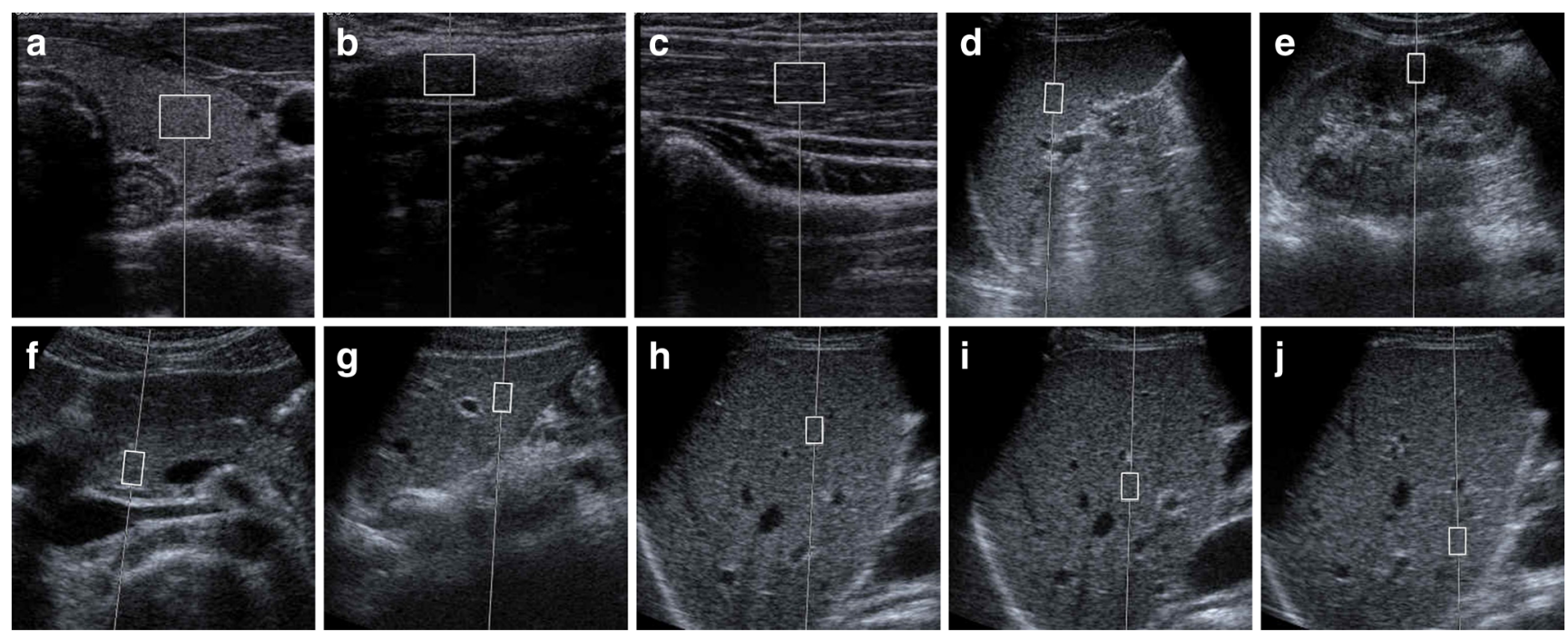

Fig. 1 ROI placement for SWS measurement in ten regions of seven organs: (a) thyroid; (b) cervical lymph node; (c) brachioradialis muscle; (d) spleen; (e) left kidney; (f) pancreas; (g) left lobe of liver; (h) right lobe of liver, $4 \mathrm{~cm}$; (i) right lobe of liver, $6 \mathrm{~cm}$; (j) right lobe of liver, $8 \mathrm{~cm}$ 
for the volunteer study $(\operatorname{ICC}(1,5))$. To assess interoperator reproducibility, the two-way random, absolute agreement, average-measure ICC was calculated using the mean SWS produced by each operator (ICC $(2,2)$ ). Measurement reliability was classified according to common criteria as excellent $(\mathrm{ICC}>0.75)$, good $(\mathrm{ICC}=0.60-0.75)$, fair $(\mathrm{ICC}=0.40-0.59)$, and poor $(\mathrm{ICC} \leq 0.40)[28]$.

A Bland-Altman (BA) plot was also used to demonstrate operator-related variations. To aid comparison across regions, the normalized difference, expressed as the percentage difference between the measurements made by each operator, i.e., $\% \Delta \mathrm{SWS}=\left(\mathrm{SWS}_{1}-\mathrm{SWS}_{2}\right) /\left(\mathrm{SWS}_{1}+\mathrm{SWS}_{2}\right) \times 100$, was calculated, where $\mathrm{SWS}_{i}$ is the mean measurement made by operator $i$. The $95 \%$ limits of agreement (LOA) between the two operators were also calculated as $1.96 \times$ the standard deviation of all measurements.

Statistical analyses were carried out using version 24.0 of the SPSS software package (SPSS).

\section{Results}

\section{Data acquisition}

In the phantom study, measurement error occurred at a depth of $1 \mathrm{~cm}$ with the 9L4 transducer and at $2 \mathrm{~cm}$ with the $4 \mathrm{C} 1$ transducer, with the failure rates being $60-100 \%$ (mean $85 \%$ ) and $55-70 \%$ (mean $62.5 \%$ ), respectively. This data was abandoned. Therefore, a total of 15 data sets (at depths of 2, 3, and $4 \mathrm{~cm}$ in five phantoms) with the 9L4 transducer and 12 data sets $(4,6$, and $8 \mathrm{~cm}$ in four phantoms) with the $4 \mathrm{C} 1$ transducer were included for analysis.

In the volunteer study, the SWSs of the thyroid, brachioradialis muscle, spleen, left kidney, pancreas, the left hepatic lobe, and right hepatic lobe at depths of 4 and $6 \mathrm{~cm}$ were measured successfully for all 30 volunteers. One volunteer had no cervical lymph node of sufficient size for SWS measurement. The long- and short-axis diameters of the other 29 cases were in the ranges $10.8-28.4 \mathrm{~mm}$ (mean $15.5 \mathrm{~mm}$ ) and $4.0-9.4 \mathrm{~mm}$ (mean $5.4 \mathrm{~mm}$ ), respectively. The failure rates for each region are shown in Table 2. Also, valid SWSs at $8 \mathrm{~cm}$ depth in the right hepatic lobe were not obtained for one volunteer. The failure rates for this patient were 100 and $50 \%$ for the two operators at a depth of $8 \mathrm{~cm}$, while the failure rates for the other 29 volunteers were in the range $0-28.6 \%$ (mean $0.8 \%$ ). Therefore, SWS in the lymph node and at a depth of $8 \mathrm{~cm}$ in the right hepatic lobe was evaluated for only 29 subjects. The measurement depth in the left lobe was in the range $2.1-6.6 \mathrm{~cm}$ (mean $4.0 \mathrm{~cm}$ ).

\section{SWS of phantoms and healthy volunteers}

The median and IQR of the SWSs for the five phantoms and volunteers are summarized in Fig. 2 and Tables 2 and 3. Note that the IQR of the phantoms demonstrates the variation of measurements in the same phantom, while the IQR for the volunteers corresponds to the variation across subjects. In addition to the cases where valid SWSs were not obtained (i.e., the measurement failure rate was too high), the extreme values found in the right hepatic lobe (corresponding to more than three times the IQR) were found for two volunteers at $4 \mathrm{~cm}$ depth, one volunteer at $6 \mathrm{~cm}$ depth, and a further three volunteers at $8 \mathrm{~cm}$ depth. All of these data were excluded from further analysis. The BMIs of the volunteers for whom either measurement failure or extreme values were observed were 25.5, 26.0, 27.2, and 29.5. Consequently, the ICC and BA plot were evaluated for only 28,29 , and 26 volunteers for the 4-, 6-, and 8-cm ROIs, respectively, in the right hepatic lobe. No extreme values were found for the other organs.

\section{Reproducibility of SWS measurement}

The intra- and interoperator ICCs for the phantom study using either five or ten measurements are summarized in Fig. 3. All ICCs were larger than 0.90 , but ICCs calculated with ten measurements were generally higher than those obtained with only five measurements. A BA plot showing the $\% \Delta$ SWS and 95\% LOA between the two operators calculated from ten measurements is presented in Fig. 4. The 95\% LOA were $17.7 \sim 15.7 \%$ for the $4 \mathrm{C} 1$ transducer and $-12.6 \sim 16.7 \%$ for the 9L4 transducer.

The intra- and interoperator reproducibilities for ten regions in seven organs are summarized in Fig. 5 and Table 4. The ICCs for intraoperator reproducibility were excellent (i.e., more than 0.75) for all regions except the pancreas, which had ICCs of 0.57 and 0.70 indicating fair to good. For the interoperator reproducibility, an excellent ICC was found only in the kidney and at a 4-cm depth in the right hepatic lobe, while values lower than 0.4 were found for the cervical lymph node and brachioradialis muscle. A BA plot for each of the ten regions is presented in the Supplemental figure, and the $\% \Delta$ SWS and $95 \%$ LOA between the two operators are summarized in Fig. 6. The 95\% LOA for the right hepatic lobe at a depth of $4 \mathrm{~cm}$ was the only one less than $25 \%$. On the other hand, the $95 \%$ LOA was $-43.5 \sim 66.9 \%$ for the kidney even though the interoperator ICC was excellent (Table 4).

\section{Discussion}

For the phantom study, the intra- and interoperator reproducibility was excellent for both transducers, which indicates high stability in measurement of a homogeneous and still phantom. Based on these results, SWS measurements using the VTQ software are highly reliable for samples under ideal conditions. This suggests that the 
Fig. 2 Box and whisker plots of the shear-wave speed for five phantoms (a) and across volunteers (b) measured by two operators using two different transducers. The phantom stiffness was measured by rheometer. Open circle $=$ outliers, asterisk $=$ extremes
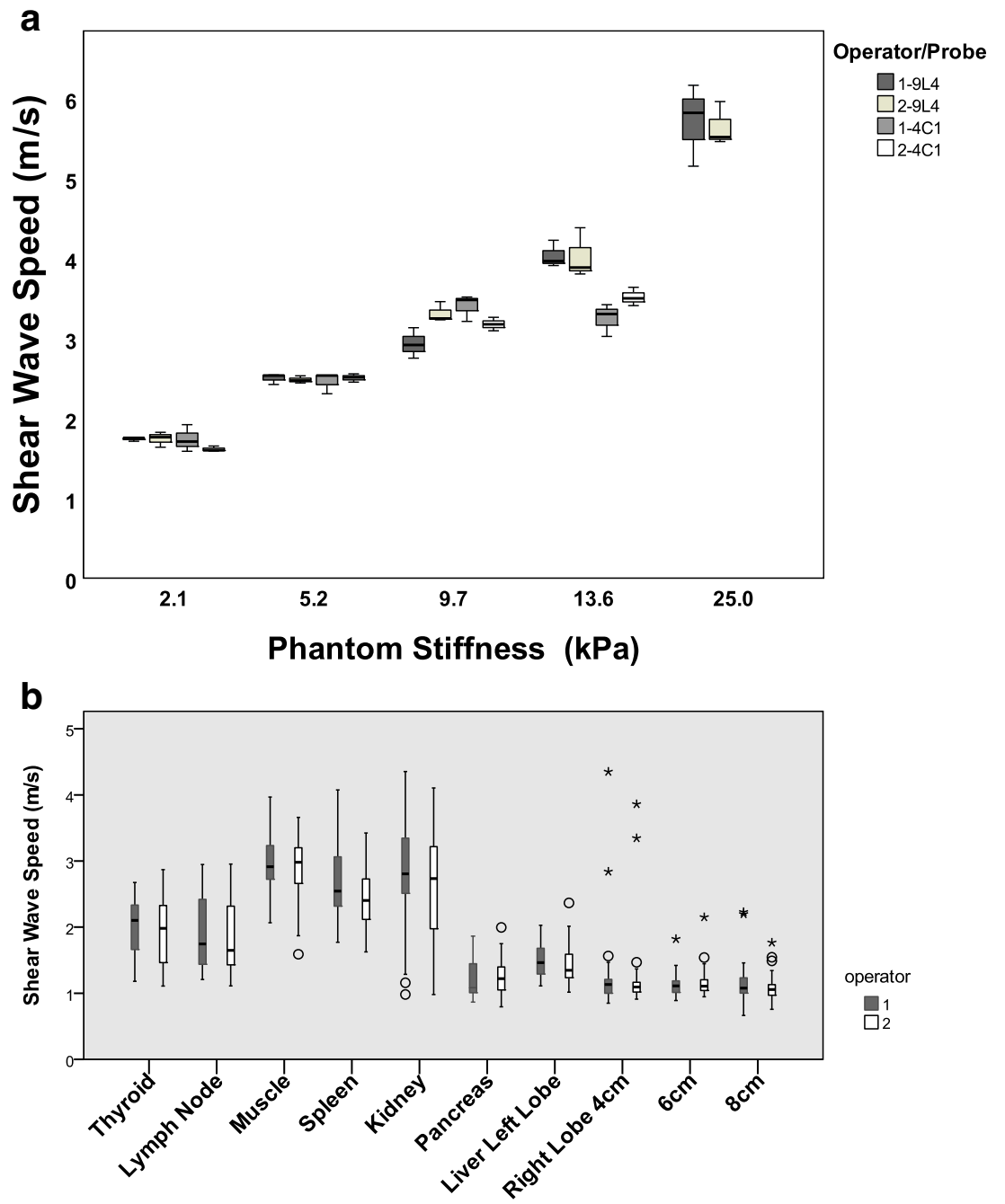

heterogeneity, movement, size of the target organ, and repeatability of placing the transducer and ROIs might be sources of insufficient reproducibility in humans.
The intraoperator reproducibility in the volunteer study was also excellent for all organs except the pancreas, for which the ICC was fair to good. The greater depth and smaller
Table 2 The number of cases, failure rate, median, and IQR of the SWS across volunteers for ten regions in seven organs

\begin{tabular}{|c|c|c|c|c|c|c|}
\hline & \multirow[t]{2}{*}{ No. of cases } & \multirow[t]{2}{*}{ Failure rate $(\%)$} & \multicolumn{2}{|c|}{ Median (m/s) } & \multicolumn{2}{|l|}{$\mathrm{IQR}(\mathrm{m} / \mathrm{s})$} \\
\hline & & & Operator 1 & Operator 2 & Operator 1 & Operator 2 \\
\hline Thyroid & 30 & $0.3[0-16.7]$ & 2.12 & 1.98 & 0.65 & 0.84 \\
\hline Lymph node & 29 & $0[0-0]$ & 1.82 & 1.65 & 0.96 & 0.86 \\
\hline Muscle & 30 & $0.3[0-16.7]$ & 2.91 & 2.97 & 0.51 & 0.54 \\
\hline Spleen & 30 & $1.0[0-28.6]$ & 2.51 & 2.39 & 0.75 & 0.59 \\
\hline Kidney & 30 & $7[0-37.5]$ & 2.81 & 2.73 & 0.82 & 1.20 \\
\hline Pancreas & 30 & $4.2[0-37.5]$ & 1.08 & 1.21 & 0.43 & 0.35 \\
\hline Liver Lt lobe & 30 & $0.3[0-16.7]$ & 1.45 & 1.36 & 0.39 & 0.34 \\
\hline Liver Rt lobe $4 \mathrm{~cm}$ & 30 & $0.8[0-50.0]$ & 1.13 & 1.10 & 0.2 & 0.14 \\
\hline Liver Rt lobe $6 \mathrm{~cm}$ & 30 & $1.4[0-37.5]$ & 1.11 & 1.11 & 0.17 & 0.15 \\
\hline Liver Rt lobe $8 \mathrm{~cm}$ & 29 & $0.8[0-28.6]$ & 1.08 & 1.06 & 0.23 & 0.16 \\
\hline
\end{tabular}

Failure rate is presented as mean [min-max]

$I Q R=$ interquartile range, $S W S=$ shear-wave speed 


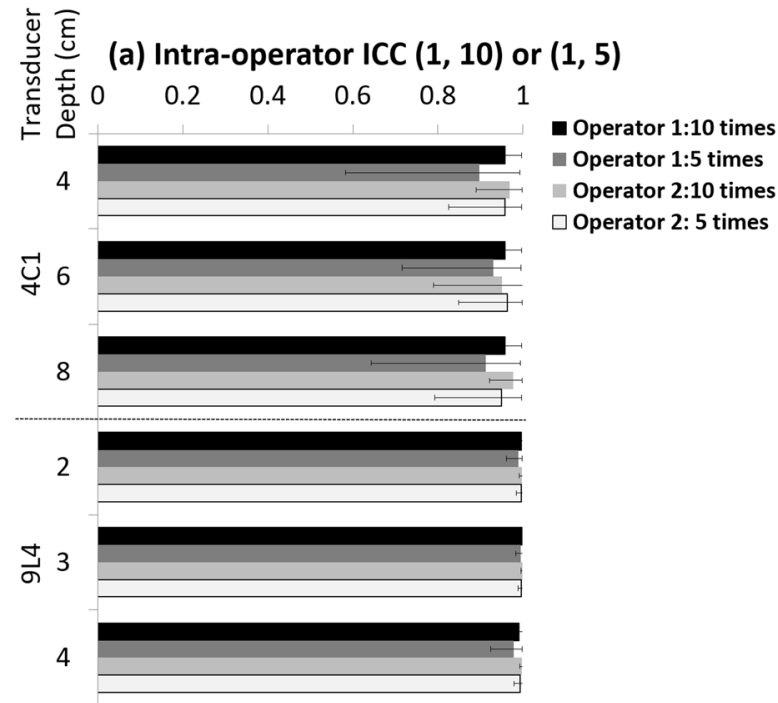

Fig. 3 Intra- and interoperator intraclass correlation coefficient (ICC) for the phantom studies. For intraoperator reproducibility (a), the one-way random, absolute agreement, average-measure $\operatorname{ICC}(1,10)$ or $\operatorname{ICC}(1,5)$

size of the pancreas in comparison to the other organs measured with the $4 \mathrm{C} 1$ transducer might be the reason for lower intraoperator reproducibility.

In contrast, the interoperator ICC was excellent only for the right hepatic lobe at $4 \mathrm{~cm}$ depth and kidney. Agreement between the two operators was high for the hepatic lobe at $4 \mathrm{~cm}$ depth as the $95 \%$ LOA was $25 \%$ or less. This result reinforces Barr and colleagues' comments that the ARFI pulse has a sweet spot at 4-5 cm depth and measurements obtained in this location may have less variability [8]. The decrease in reproducibility with measurement depth in our results is consistent with a previous study measuring focal liver lesions [29].

\section{(a) 4C1 transducer}

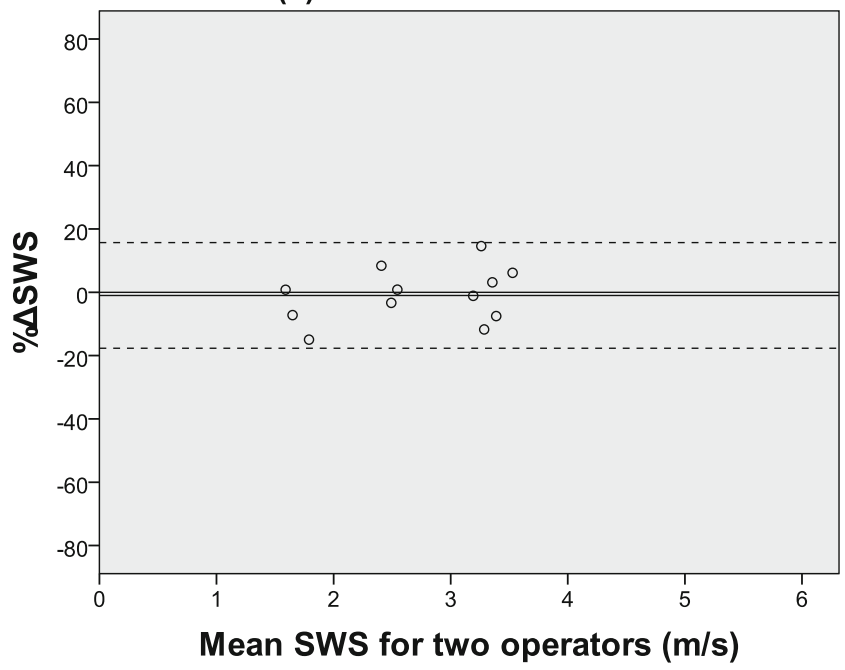

Fig. 4 Bland-Altman plot for the interoperator reproducibility of SWS measurement in phantoms with a 4C1 transducer (a) and a 9L4 transducer (b). The $X$-axis corresponds to the average SWS for two operators and the

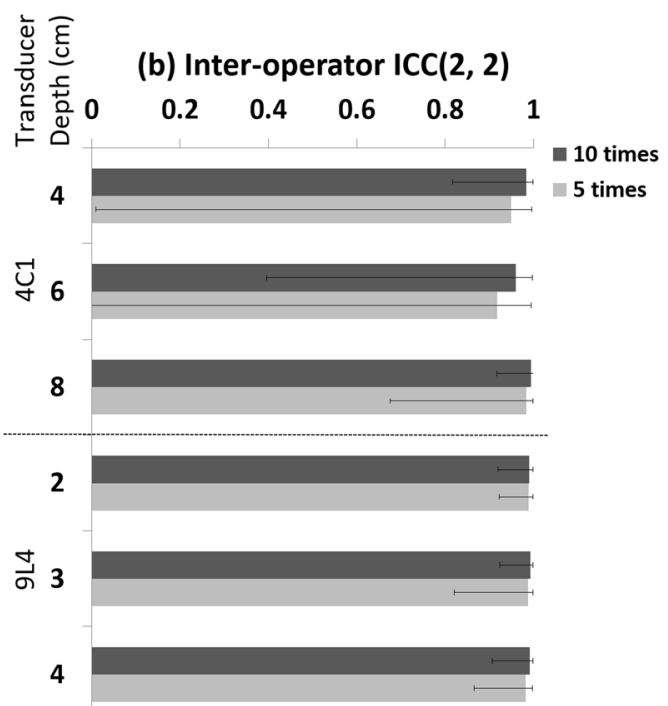

was calculated, and for interoperator reproducibility $\mathbf{b}$, the two-way random, absolute agreement, average-measure $\operatorname{ICC}(2,2)$ was calculated

For the kidney, on the other hand, the $95 \%$ LOA was as large as $-43.5 \sim 66.9 \%$ despite an excellent interoperator ICC. This discrepancy between the $95 \%$ LOA and interoperator ICC in the kidney is thought to be due to the inhomogeneity of the renal parenchyma consisting of the cortex and medulla [30]. Reproducibility depends on both the magnitude of measurement error as well as the true heterogeneity in the population from which measurements are made [31]. The wide variability of SWS in the kidney in our study is similar to that measured in previous studies [17, 30, 32, 33].

In our study, the interoperator ICC was as poor as 0.4 or less for cervical lymph node and brachioradialis muscle

(b) 9 L4 transducer

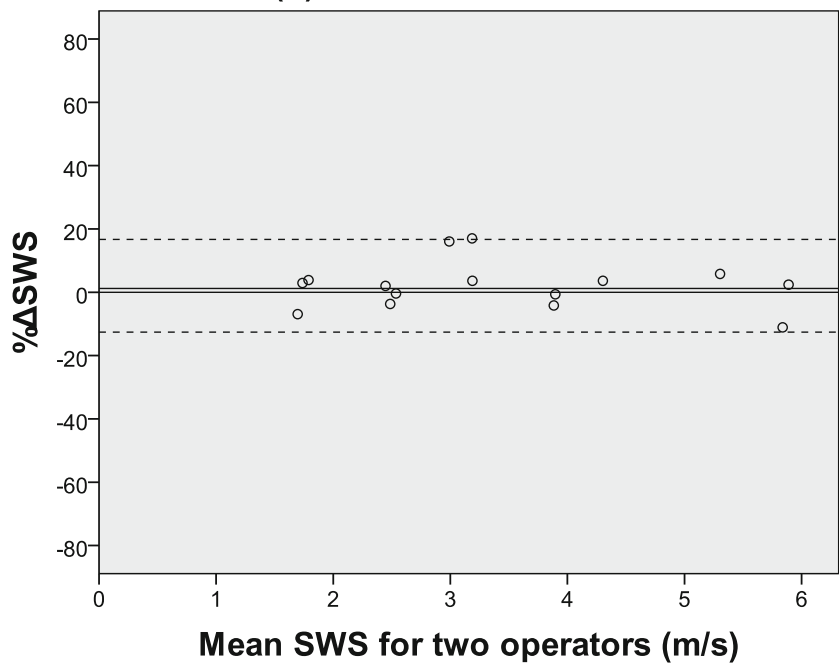

$Y$-axis is $\% \Delta$ SWS. SWS $=$ shear-wave speed, solid line $=$ mean bias, dashed line $=95 \%$ limits of agreement $(1.96 \times$ standard deviation $)$ 
(a) Intra-operator ICC $(1,5)$

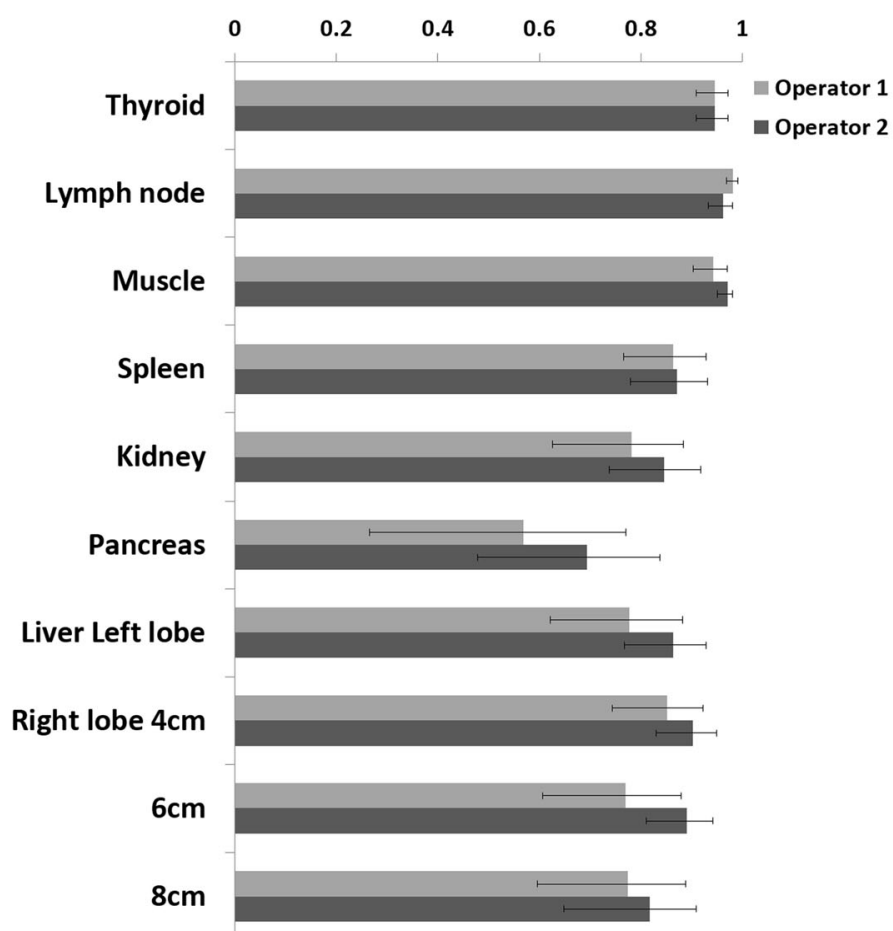

Fig. 5 Intra- and interoperator intraclass correlation coefficient (ICC) for ten regions in seven organs. For intraoperator reproducibility (a), the oneway random, absolute agreement, average-measure $\operatorname{ICC}(1,5)$ was

despite an excellent intraoperator ICC of more than 0.9. The size of the lymph nodes was generally small because the subjects were healthy volunteers. The small size of the lymph node may produce subtle discordance of ROI positioning between the two operators and, hence, differences in SWS measurement and low interoperator reproducibility.

It is reported that the measurement plane and the angle of the transducer relative to the muscle might affect the reproducibility of measurements due to anisotropy of the tissue [34]. In our study, the two operators placed the transducer perpendicular to the body surface along the longitudinal axis of the brachioradialis muscle, so the effect of anisotropy is thought to be small. For the brachioradialis muscle, the small IQR of the SWS estimates is a possible reason for the low interoperator ICC. When analyzing measurement reliability, we suggest that investigators report estimates of within-population IQR and $95 \%$ LOA, in addition to the ICC.

There are several studies discussing the reproducibility of SWS measurement in the liver. Our estimate of the intraoperator ICC for the right hepatic lobe at $4 \mathrm{~cm}$ depth was similar to that of previous studies using ARFI [12, 35, 36], but our interoperator ICC was worse than that in the results of Woo et al [12] where SWS was measured nine times. Some studies $[37,38]$ have shown that five SWE measurements could be used instead of ten without a significant effect

\section{(b) Inter-operator $\operatorname{ICC}(2,2)$}

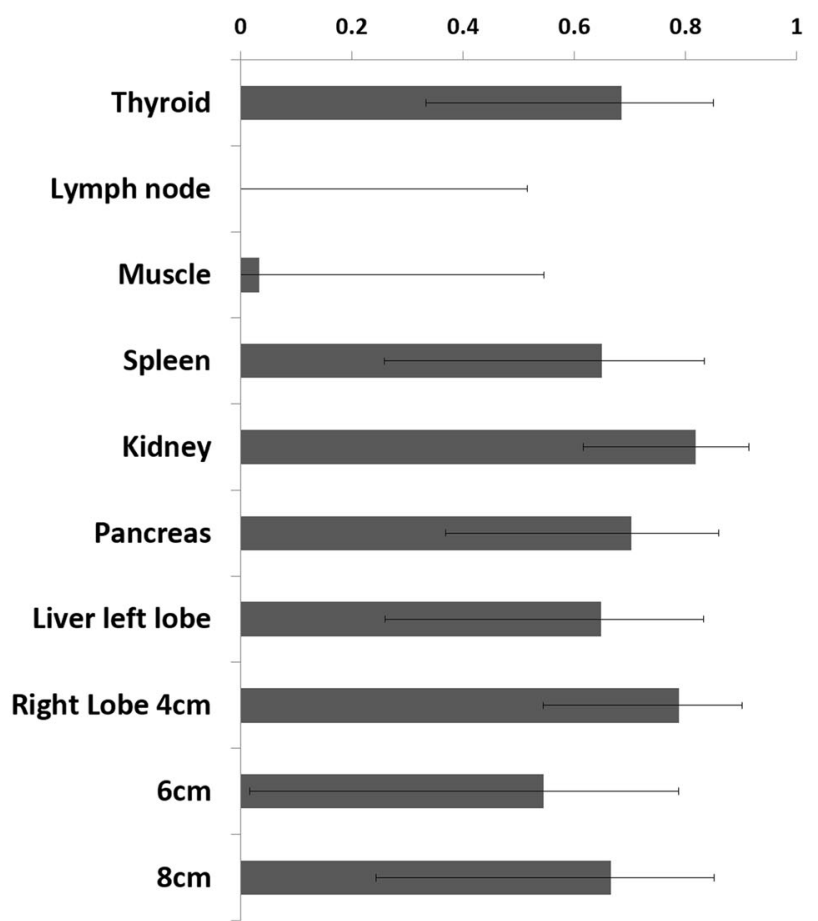

calculated, and for interoperator reproducibility (b), the two-way random, absolute agreement, average-measure $\operatorname{ICC}(2,2)$ was calculated

on diagnostic performance. Other studies [39, 40] have suggested that the optimal minimum number of measurements required was six. Based on the results of our phantom study, where ICCs with ten measurements were higher than those with five measurements, it appears that more measurements may lead to higher stability. Our subjects were healthy volunteers so that the variability is thought to be smaller than that of studies including patients with hepatic disease. As mentioned earlier, as the reliability of a measurement method depends

Table 3 Median and IQR for the SWS of five phantoms

\begin{tabular}{lllllll}
\hline & \multirow{2}{*}{ Probe } & \multicolumn{2}{l}{ Median $(\mathrm{m} / \mathrm{s})$} & & \multicolumn{2}{l}{$\mathrm{IQR}(\mathrm{m} / \mathrm{s})$} \\
\cline { 3 - 4 } \cline { 6 - 7 } \cline { 6 - 7 } & & Operator 1 & Operator 2 & & Operator 1 & Operator 2 \\
\hline \multirow{2}{*}{$2.1 \mathrm{kPa}$} & $9 \mathrm{~L} 4$ & 1.76 & 1.76 & & 0.02 & 0.09 \\
& $4 \mathrm{C} 1$ & 1.71 & 1.60 & & 0.17 & 0.03 \\
$5.2 \mathrm{kPa}$ & $9 \mathrm{~L} 4$ & 2.53 & 2.47 & & 0.06 & 0.05 \\
& $4 \mathrm{C} 1$ & 2.53 & 2.51 & & 0.11 & 0.05 \\
$9.7 \mathrm{kPa}$ & $9 \mathrm{~L} 4$ & 2.92 & 3.25 & & 0.19 & 0.11 \\
& $4 \mathrm{C} 1$ & 3.48 & 3.17 & & 0.15 & 0.08 \\
$13.3 \mathrm{kPa}$ & $9 \mathrm{~L} 4$ & 3.97 & 3.77 & & 0.16 & 0.29 \\
& $4 \mathrm{C} 1$ & 3.30 & 3.50 & & 0.20 & 0.11 \\
$25.0 \mathrm{kPa}$ & $9 \mathrm{~L} 4$ & 5.82 & 5.51 & & 0.51 & 0.25 \\
\hline
\end{tabular}




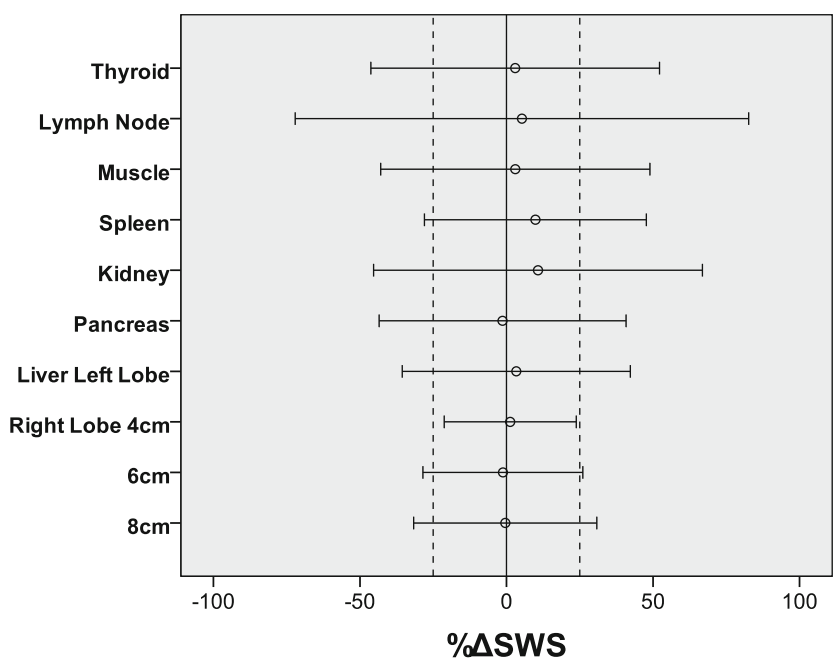

Fig. 6 The $\% \Delta$ SWS between two operators relative to their mean and $95 \%$ limits of agreement. SWS $=$ shear-wave speed, dashed line $=25 \%$. $\% \Delta \mathrm{SWS}=\left(\mathrm{SWS}_{1}-\mathrm{SWS}_{2}\right) /\left(\mathrm{SWS}_{1}+\mathrm{SWS}_{2}\right) \times 100$, where $\mathrm{SWS}_{i}$ is the mean measurement made by operator $\mathrm{i}$

upon the heterogeneity of the population, a direct comparison of the ICC between different populations does not make much sense. In the phantom study, measurement error often occurred for the shallowest ROI $(1 \mathrm{~cm}$ for the 9L4 transducer and $2 \mathrm{~cm}$ for the $4 \mathrm{C} 1$ transducer), and thus, those data were abandoned. The measurement error is thought to be due to multiple reflections from the surface of the phantom because the phantoms were wrapped in plastic film to prevent drying
[27]. For the volunteer study, the volunteers for whom measurement failure occurred had BMIs of 25.5-29.5, which were four of the top 5 BMIs in the group. The measurement failure is thought to be due to corpulence [41].

Our study has several limitations. We assessed intraoperator reproducibility only within one session of measurement. Ideally, the reproducibility of measurement should also be examined in another session on the same day or the following day (e.g., intraday or interday reproducibility for the same operator), in order to estimate the variation when the same operator repeats the examination. We only performed measurements on normal organ parenchyma. Reproducibility in the examination of tumors, cirrhosis, and other diseases may be different.

We evaluated reproducibility for only one application with one device at one facility. For SWS to be used widely as a reliable biomarker, it would be beneficial to evaluate agreement between different applications, multiple devices, and multiple facilities [42].

In conclusion, our phantom study indicated the high reliability of SWS measurements with pSWE under ideal conditions. For the volunteer study, the intraoperator reproducibility was also generally high, but the interoperator reproducibility from only five measurements was not high enough except for the right hepatic lobe at $4 \mathrm{~cm}$ depth. To evaluate interoperator reproducibility, the 95\% LOA between operators should be considered in addition to the ICC. It is a great merit of pSWE to be able to evaluate the stiffness quantitatively with
Table 4 Intra- and interoperator reproducibilities across volunteers for ten regions in seven organs

\begin{tabular}{|c|c|c|c|c|c|c|}
\hline & \multirow[t]{2}{*}{$\mathrm{MHz}$} & \multicolumn{2}{|l|}{ Intraoperator ICC } & \multicolumn{3}{|c|}{ Interoperator reproducibility } \\
\hline & & Operator 1 & Operator 2 & ICC & $\begin{array}{l}\text { Mean } \\
\text { bias (\%) }\end{array}$ & $\begin{array}{l}95 \% \text { LOA } \\
(\%)\end{array}$ \\
\hline Thyroid & 9 & $0.95[0.91-0.97]$ & $0.95[0.91-0.97]$ & $0.69[0.33-0.85]$ & 2.96 & $-46.0 \sim 52.2$ \\
\hline $\begin{array}{r}\text { Lymph } \\
\text { node }\end{array}$ & 9 & $0.98[0.97-0.99]$ & $0.96[0.99-0.98]$ & $0[0-0.52]$ & 5.27 & $-72.1 \sim 82.6$ \\
\hline Muscle & 9 & $.94[.90-.97]$ & $0.97[0.95-0.98]$ & $0[0-0.55]$ & 3 & $-42.9 \sim 48.9$ \\
\hline Spleen & 4 & $0.86[0.77-0.93]$ & $0.87[0.78-0.93]$ & $0.65[0.26-0.83]$ & 9.87 & $-28.0 \sim 47.7$ \\
\hline Kidney & 4 & $0.78[0.63-0.88]$ & $0.85[0.74-0.92]$ & $0.82[0.62-0.91]$ & 10.8 & $-45.3 \sim 66.9$ \\
\hline Pancreas & 4 & $0.57[0.27-0.77]$ & $0.69[0.48-0.84]$ & $0.70[0.37-0.86]$ & -1.35 & $-43.8 \sim 40.8$ \\
\hline $\begin{array}{c}\text { Liver Lt } \\
\text { lobe }\end{array}$ & 4 & $0.78[0.62-0.88]$ & $0.86[0.77-0.93]$ & $0.65[0.26-0.83]$ & 3.34 & $-35.6 \sim 42.2$ \\
\hline $\begin{array}{c}\text { Liver Rt } \\
\text { lobe } \\
4 \mathrm{~cm}\end{array}$ & 4 & $0.85[0.74-0.92]$ & $0.90[0.83-0.95]$ & $0.79[0.54-0.90]$ & 1.26 & $-21.3 \sim 23.8$ \\
\hline $\begin{array}{c}\text { Liver Rt } \\
\text { lobe } \\
6 \mathrm{~cm}\end{array}$ & 4 & $0.77[0.61-0.88]$ & $0.89[0.81-0.94]$ & $0.55[0.02-0.79]$ & -1.21 & $-29.0 \sim 25.6$ \\
\hline $\begin{array}{c}\text { Liver Rt } \\
\text { lobe } \\
8 \mathrm{~cm}\end{array}$ & 4 & $0.77[0.60-0.89]$ & $0.82[0.68-0.91]$ & $0.67[0.24-0.85]$ & -0.41 & $-31.7 \sim 30.8$ \\
\hline
\end{tabular}

Data in parentheses are $95 \%$ confidence intervals

$I C C=$ intraclass correlation coefficient, $L O A=$ limits of agreement 
ease. To reliably utilize this measurement method clinically, it is essential to have a strong understanding of the degree of reproducibility.

Funding The authors state that this work has not received any funding.

\section{Compliance with ethical standards}

Guarantor The scientific guarantor of this publication is Riwa Kishimoto.

Conflict of interest The authors declare that they have no conflict of interest.

Statistics and biometry One of the authors has significant statistical expertise.

Informed consent Written informed consent was obtained from all volunteers in this study.

Ethical approval Institutional Review Board approval was obtained.

Methodology

- prospective

- experimental

- performed at one institution

Open Access This article is distributed under the terms of the Creative Commons Attribution 4.0 International License (http:// creativecommons.org/licenses/by/4.0/), which permits unrestricted use, distribution, and reproduction in any medium, provided you give appropriate credit to the original author(s) and the source, provide a link to the Creative Commons license, and indicate if changes were made.

\section{References}

1. Ferraioli G, Filice C, Castera L et al (2015) WFUMB guidelines and recommendations for clinical use of ultrasound elastography: part 3: liver. Ultrasound Med Biol 41(5):1161-1179

2. Barr RG, Nakashima K, Amy D et al (2015) WFUMB guidelines and recommendations for clinical use of ultrasound elastography: part 2: breast. Ultrasound Med Biol 41(5):1148-1160

3. Cosgrove D, Barr R, Bojunga J et al (2017) WFUMB guidelines and recommendations on the clinical use of ultrasound elastography: part 4. Thyroid. Ultrasound Med Biol 43(1):4-26

4. Barr RG, Cosgrove D, Brock M et al (2017) WFUMB guidelines and recommendations on the clinical use of ultrasound elastography: part 5. Prostate. Ultrasound Med Biol 43(1):27-48

5. Shiina T, Nightingale KR, Palmeri ML et al (2015) WFUMB guidelines and recommendations for clinical use of ultrasound elastography: part 1: basic principles and terminology. Ultrasound Med Biol 41(5):1126-1147

6. Palmeri M, Nightingale KR, Fielding S et al (2015) RSNA QIBA ultrasound shear wave speed phase II phantom study in viscoelastic media. IEEE International Ultrasonic Symposium. https://oi.org/ 10.1109/ULTSYM.2015.0283
7. Sandrin L, Fourquet B, Hasquenoph JM et al (2003) Transient elastography: a new noninvasive method for assessment of hepatic fibrosis. Ultrasound Med Biol 29(12):1705-1713

8. Barr RG, Ferraioli G, Palmeri ML et al (2015) Elastography assessment of liver fibrosis: Society of Radiologists in ultrasound consensus conference statement. Radiology 276(3):845-861

9. Tang A, Cloutier G, Szeverenyi NM, Sirlin CB (2015) Ultrasound elastography and MR elastography for assessing liver fibrosis: part 1, principles and techniques. AJR Am J Roentgenol 205(1):22-32

10. Bercoff J, Tanter M, Muller M, Fink M (2004) The role of viscosity in the impulse diffraction field of elastic waves induced by the acoustic radiation force. IEEE Trans Ultrason Ferroelectr Freq Control 51(11):1523-1536

11. Friedrich-Rust M, Wunder K, Kriener S et al (2009) Liver fibrosis in viral hepatitis: noninvasive assessment with acoustic radiation force impulse imaging versus transient elastography. Radiology 252(2):595-604

12. Woo H, Lee JY, Yoon JH, Kim W, Cho B, Choi BI (2015) Comparison of the reliability of acoustic radiation force impulse imaging and supersonic shear imaging in measurement of liver stiffness. Radiology 277(3):881-886

13. Van Beers BE, Daire JL, Garteiser P (2015) New imaging techniques for liver diseases. J Hepatol 62(3):690-700

14. Tozaki M, Saito M, Benson J, Fan L, Isobe S (2013) Shear wave velocity measurements for differential diagnosis of solid breast masses: a comparison between virtual touch quantification and virtual touch IQ. Ultrasound Med Biol 39(12):2233-2245

15. Samir AE, Dhyani M, Anvari A et al (2015) Shear-wave elastography for the preoperative risk stratification of follicularpatterned lesions of the thyroid: diagnostic accuracy and optimal measurement plane. Radiology 277(2):565-573

16. Kawada N, Tanaka S, Uehara H et al (2014) Potential use of point shear wave elastography for the pancreas: a single center prospective study. Eur J Radiol 83(4):620-624

17. Guo LH, Xu HX, Fu HJ, Peng A, Zhang YF, Liu LN (2013) Acoustic radiation force impulse imaging for noninvasive evaluation of renal parenchyma elasticity: preliminary findings. PLoS One 8(7): 68925

18. Lu Q, Wen JX, Huang BJ, Xue LY, Wang WP (2015) Virtual touch quantification using acoustic radiation force impulse (ARFI) technology for the evaluation of focal solid renal lesions: preliminary findings. Clin Radiol 70(12):1376-1381

19. Alan B, Utangac M, Goya C, Daggulli M (2016) Role of acoustic radiation force impulse (ARFI) elastography in determination of severity of benign prostate hyperplasia. Med Sci Monit 22:45234528

20. Fujiwara T, Tomokuni J, Iwanaga K, Ooba S, Haji T (2013) Acoustic radiation force impulse imaging for reactive and malignant/metastatic cervical lymph nodes. Ultrasound Med Biol 39(7):1178-1183

21. Rosskopf AB, Ehrmann C, Buck FM, Gerber C, Fluck M, Pfirrmann CW (2016) Quantitative shear-wave US elastography of the supraspinatus muscle: reliability of the method and relation to tendon integrity and muscle quality. Radiology 278(2):465-474

22. Suda T, Okawa O, Masaoka R et al (2017) Shear wave elastography in hepatitis $\mathrm{C}$ patients before and after antiviral therapy. World $\mathrm{J}$ Hepatol 9(1):64-68

23. Ma Y, Zhang S, Li J, Li J, Kang Y, Ren W (2017) Comparison of strain and shear-wave ultrasounic elastography in predicting the pathological response to neoadjuvant chemotherapy in breast cancers. Eur Radiol 27(6):2282-2291

24. Ahn SJ, Lee JM, Chang W et al (2017) Prospective validation of intra- and interobserver reproducibility of a new point shear wave elastographic technique for assessing liver stiffness in patients with chronic liver disease. Korean J Radiol 18(6):926-935 
25. Cosgrove DO, Berg WA, Doré CJ et al (2012) BE1 Study Group. Shear wave elastography for breast masses is highly reproducible. Eur Radiol 22(5):1023-1032

26. Anvari A, Dhyani M, Stephen AE, Samir AE (2016) Reliability of shear-wave elastography estimates of the Young modulus of tissue in follicular thyroid neoplasms. Am J Roentgenol 206(3):609-1627

27. Kishimoto R, Suga M, Koyama A, et al (2017) Measuring shearwave speed with point shear-wave elastography and MR elastography: a phantom study. BMJ Open 7(1):e013925

28. Vetter NC, Steding J, Jurk S, Ripke S, Mennigen E, Smolka MN (2017) Reliability in adolescent fMRI within two years - a comparison of three tasks. Sci Rep 7(1):2287

29. Park HS, Kim YJ, Yu MH, Jung SI, Jeon HJ (2015) Shear wave elastography of focal liver lesion: intraobserver reproducibility and elasticity characterization. Ultrasound Q 31(4):262-271

30. Marticorena Garcia SR, Grossmann M, Lang ST et al (2018) Fullfield-of-view time-harmonic elastography of the native kidney. Ultrasound Med Biol 44(5):949-954

31. Bartlett JW, Frost C (2008) Reliability, repeatability and reproducibility: analysis of measurement errors in continuous variables. Ultrasound Obstet Gynecol 31(4):466-475

32. Goertz RS, Amann K, Heide R, Bernatik T, Neurath MF, Strobel D (2011) An abdominal and thyroid status with acoustic radiation force impulse elastometry - a feasibility study: acoustic radiation force impulse elastometry of human organs. Eur J Radiol 80(3): e226-e230

33. Bob F, Bota S, Sporea I, Sirli R, Petrica L, Schiller A (2014) Kidney shear wave speed values in subjects with and without renal pathology and inter-operator reproducibility of acoustic radiation force impulse elastography (ARFI) - preliminary results. PLoS One 9(11):e113761

34. Cortez CD, Hermitte L, Ramain A, Mesmann C, Lefort T, Pialat JB (2016) Ultrasound shear wave velocity in skeletal muscle: a reproducibility study. Diagn Interv Imaging 97(1):71-79

35. Balakrishnan M, Souza F, Munoz C et al (2016) Liver and spleen stiffness measurements by point shear wave elastography via acoustic radiation force impulse: intraobserver and interobserver variability and predictors of variability in a US population. J Ultrasound Med 35(11):2373-2380

36. Bota S, Sporea I, Sirli R, Popescu A, Danila M, Costachescu D (2012) Intra- and interoperator reproducibility of acoustic radiation force impulse (ARFI) elastography_preliminary results. Ultrasound Med Biol 38(7):1103-1108

37. Choi SH, Jeong WK, Kim Y et al (2016) How many times should we repeat measuring liver stiffness using shear wave elastography? 5-repetition versus 10-repetition protocols. Ultrasonics 72:158-164

38. Ferraioli G, Maiocchi L, Lissandrin R, Tinelli C, De Silvestri A, Filice C (2016) Accuracy of the Elast PQ technique for the assessment of liver fibrosis in patients with chronic hepatitis C: a "real life" single center study. J Gastrointestin Liver Dis 25(3):331-335

39. Yoon JH, Lee JM, Han JK, Choi BI (2014) Shear wave elastography for liver stiffness measurement in clinical sonographic examinations: evaluation of intraobserver reproducibility, technical failure, and unreliable stiffness measurements. J Ultrasound Med 33(3):437-447

40. Fang C, Jaffer OS, Yusuf GT et al (2018) Reducing the number of measurements in liver point shear-wave elastography: factors that influence the number and reliability of measurements in assessment of liver fibrosis in clinical practice. Radiology 287(3):844-852

41. Trout AT, Dillman JR, Xanthakos S et al (2016) Prospective assessment of correlation between US acoustic radiation force impulse and MR elastography in a pediatric population: dispersion of US shear-wave speed measurement matters. Radiology 281(2):544552

42. Dillman JR, Chen S, Davenport MS et al (2015) Superficial ultrasound shear wave speed measurements in soft and hard elasticity phantoms: repeatability and reproducibility using two ultrasound systems. Pediatr Radiol 45(3):376-385

Publisher's note Springer Nature remains neutral with regard to jurisdictional claims in published maps and institutional affiliations. 